This is an electronic reprint of the original article. This reprint may differ from the original in pagination and typographic detail.

Author(s): Hytönen, Vesa; Puchko, Oleksandr; Höhne, Thomas; Chapman, Thomas

Title: $\quad$ Introduction of multiflow for HSDPA

Year: $\quad 2012$

Version:

Please cite the original version:

Hytönen, V., Puchko, O., Höhne, T., \& Chapman, T. (2012). Introduction of multiflow for HSDPA. In A. Levi, M. Badra, M. Cesana, M. Ghassemian, Ö. Gürbüz, N. Jabeur, M. Klonowski, A. Maña, S. Sargento, \& S. Zeadally (Eds.), 2012 5th International Conference on New Technologies, Mobility and Security (NTMS). IEEE.

https://doi.org/10.1109/NTMS.2012.6208684

All material supplied via JYX is protected by copyright and other intellectual property rights, and duplication or sale of all or part of any of the repository collections is not permitted, except that material may be duplicated by you for your research use or educational purposes in electronic or print form. You must obtain permission for any other use. Electronic or print copies may not be offered, whether for sale or otherwise to anyone who is not an authorised user. 


\section{Introduction of Multiflow for HSDPA}

\author{
Vesa Hytönen, Oleksandr Puchko \\ Department of Mathematical Information Technology, \\ P.O.Box 35 (Agora), FI-40014, \\ University of Jyväskylä, Finland \\ \{vesa.a.hytonen, oleksandr.puchko\}@jyu.fi
}

\author{
Thomas Höhne \\ CEF CTO Research,
} Roke Manor Research Ltd., Nokia Siemens Networks, Espoo, Finland thomas.hoehne@nsn.com

\begin{abstract}
This paper introduces a multi-cell transmission scheme for High-Speed Downlink Packet Access (HSDPA) networks, called Multiflow. In this concept, downlink data is transmitted to a user terminal at the border of two cells from one or both of the cells. The cells may belong to same NodeB or to two different NodeBs. The data flows are separated by different scrambling codes used by each associated cell, thus the flows can be treated independently. This provides increased multi-user diversity by means of flexibility in downlink resource management, in addition to the spatial diversity of multiple transmission locations. Another important gain mechanism for this scheme is realized by short-term load balancing between neighboring cells. We evaluate the performance of Multiflow by semi-static system level simulations. The presented results reveal high throughput gains for users in handover areas and also a slight improvement to overall network performance.

Index Terms-Multipoint transmission, HSDPA.
\end{abstract}

\section{INTRODUCTION}

Satisfying the growing requirements of mobile networking customers, telecommunication companies and service providers are seeking new ways to improve the capacity of their networks. Enhancements for WCDMA based HSDPA are especially of interest for operators as the next generation mobile networks, such as LTE, are just starting to be rolled out, whereas HSPA has already been deployed worldwide. Over time, the evolvement of the networks has changed from providing increased peak data rates to focusing more on serving the customers with improved fairness. The concept discussed in this paper is well positioned to do so as it is applicable to all cell-edge users and is able to improve their throughput and thereby user experience in low to medium load scenarios.

The family of Multiflow schemes for HSDPA has been approved as a 3GPP work item ([1]). There, the traffic is forwarded to the User Equipment (UE) from two sectors controlled by the same or different NodeBs. In Multiflow, downlink transport blocks containing different data can be transmitted independently, at different times or simultaneously, from two cells over separate flows. As the concurrent flows are not orthogonal, an interference aware receiver is mandatory at the UE side. By the usage of two data streams UE throughput could be, in theory, doubled. True gain levels are however lower due to channel imbalance between the cells and suboptimal interference cancellation at the UE. In this study the focus is on Multiflow applied in single-carrier network, where all the cells share a common carrier frequency, and transmissions are separated by dedicated scrambling codes for each cell.

Due to its novelty, literature about Multiflow other than 3 GPP study item reports is scarce. However, multipoint transmission concepts have not been studied only for HSDPA. A considerable amount of research has been done for Coordinated Multipoint Transmission (CoMP) in LTE-Advanced (LTE-A). In LTE-A, as in HSDPA, the main reason for utilizing multi-cell transmission schemes is to elevate cell edge and possibly system throughput. There are two sub-schemes of CoMP in downlink: Joint Processing and Coordinated Scheduling/Coordinated beamforming (CS/CB) [2]. Further, Joint Processing can be divided into two categories. First, so called Dynamic Cell Selection where data is transmitted from one eNodeB at a time [3]. This technique is quite similar to Single-Frequency Dual-Cell (SF-DC) Switching in HSDPA, one of the multi-cell transmission schemes evaluated during the study item phase of the current work item. In the second category, Joint Transmission, downlink transmission occurs from multiple eNodeBs to a single UE simultaneously, thus having strong resemblance with Multiflow scheme studied in this paper [4].

The rest of the document is organized as follows. Section II gives an overview of Multiflow describing the gain mechanisms and requirements for the deployment. In Section III the simulator environment together with the achieved results are discussed. Section IV concludes the paper.

\section{THE CONCEPT}

Users at the cell borders are usually subject to low signal level due to strong path loss caused by long distance to NodeB or a location that is not in the cell's antenna beam direction. They also suffer from high interference from other sites in the network. As the center cell terminals enjoy good signal quality, the overall fairness in terms of user experience in the network might be low. This can be corrected by the scheduler but at a cost of cell capacity.

Multiflow will help cell edge UEs as they are able to receive data from two transmitting cells, located either in the same site or different sites. Depending on the scheduler employed this can be achieved by using resources of the neighbor cell which would otherwise be used there for cell-center users, or by restricting assisting transmissions from the neighbor 
cell to TTIs where the neighboring cell has free resources, thereby enabling short-term load-balancing. In theory, the cell edge UEs utilizing two transmissions may even double their throughput if the channel conditions to both cells are in balance.

Assuming that no scheduling coordination between the transmitting cells is established, data blocks can be transmitted from two cells to a single user concurrently and they will present interference to each other at the UE. In order to mitigate the interference, a type $3 \mathrm{i}$ receiver with antenna diversity is required in the user equipment which provides interference cancellation with a chip level equalizer. This type of receiver calculates an equalizer filter that maximizes the overall SINR by using the knowledge of the colored interference of neighboring transmissions [5].

\section{A. Gain mechanisms}

The gain mechanism for UEs in the network with enabled Multiflow is two-fold. First, utilization of the second data stream increases per-UE data rates. The amount of extra throughput depends on the post-receiver SINR of both links and load levels of the cells. Second, Multiflow directly helps users at the cell borders by which they are able to finish their data transfer events faster, offering increased opportunities to resource utilization for other users in the network. Since celledge users usually remain in the network long due to high signal losses and inter-cell interference, reduction of their activity time in the network improves the throughput fairness across the UEs in the system. As demonstrated later this can be achieved without a negative impact on cell-center users.

A Multiflow capable UE may be allocated resources from both cells in Multiflow active set independently which thereupon increases the utilization rate of low load cells while enhancing scheduling opportunities for UEs in highly loaded cells.

\section{B. Flow control and signaling}

In Multiflow, the source data packets are divided onto separate data flows, routed over separate NodeBs and received (potentially) in parallel by the UE. Once the data packets have been decoded successfully, they will be re-ordered and delivered to higher layers. The data can be split at different layers in the network or NodeB. The best way of doing so is an active area of research.

Intra-site Multiflow enables the use of fast data flow split on MAC-ehs layer at the NodeB, whereas for inter-site operation the split needs to be performed on the PDCP or RLC layer in the Radio Network Controller (RNC), see Fig. 1. In HSDPA, there is no direct link for communication between the NodeBs, thus the split operation in RNC is the only option for inter-site NodeBs, if a possible core network operation is excluded [6].

At the receiver side, two MAC-ehs entities are needed for the RLC PDU reception of the primary and secondary transmission, in case of inter-site Multiflow. Similarly, one MAC-ehs entity is sufficient in case only intra-site Multiflow is supported by the network.
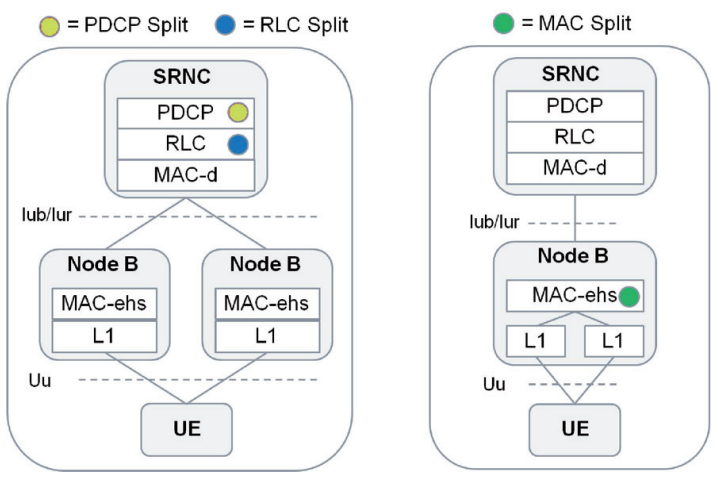

Fig. 1. Data flow split options for Multiflow

The Radio Network Controller (RNC) is responsible for enabling and disabling Multiflow on user-by-user basis. It informs the UEs which cells they should monitor for transmission and consequently provide Channel Quality Indication (CQI) feedback messages for. The CQI operation in Multiflow would closely follow that of Dual-Cell HSDPA and HSDPA MIMO, where several CQIs are reported to the nodeB per TTI [7]. Likewise, for Multiflow the amount of CQIs transmitted in UL would be adjusted according to the number of configured carriers in DL. As with existing schemes, the feedback may be reported in compound messages or in time-multiplexed fashion (see 3GPP Technical Specification 25.212, Table 15C.4). Similar to CQI signaling, also the Hybrid Automatic Repeat Request (HARQ) acknowledgments need to be reported in UL for all flows.

In addition to selecting which UEs should enable or disable Multiflow support, the RNC decides which sectors in the network may participate the multi-cell transmission operations. However, it is a matter of NodeB scheduler to dynamically decide based on the feedback messages which cells from those selected by the RNC may apply a Multiflow transmission on each TTI. As for the other operations, additional higher layer (L2, L3) signaling will support the activation of Multiflow as well as data flow control [8].

\section{SYSTEM MODEL}

A semi-static cell-based network simulator is used to model Multiflow in an HSDPA environment. The model introduces 57 NodeBs, each having three sectors covering 120 degree angles. Simulation parameters are introduced in Table I. They follow the assumptions defined by 3GPP standardization [9]. Both inter- and intra-site Multiflow operation are enabled, applying dual data flow possibility for users located at each cell border where the signal strength imbalance between two cells falls within $6 \mathrm{~dB}$.

During a simulation UEs are stationary. However, fast fading is introduced to produce channel variation. Shadowing is modeled by running several simulation sets with different seeding where UEs are dropped randomly in the simulation 
TABLE I

SIMULATION PARAMETERS

\begin{tabular}{|c|c|}
\hline Parameter & Value \\
\hline Cell Layout & $\begin{array}{l}\text { Hexagonal grid, } 19 \text { NodeBs, } 3 \text { sec- } \\
\text { tors per NodeB with wrap-around }\end{array}$ \\
\hline Inter-site distance & $1000 \mathrm{~m}$ \\
\hline Carrier Frequency & $2000 \mathrm{MHz}$ \\
\hline Path Loss & $\begin{array}{l}\mathrm{L}=128.1+37.6 \log 10(\mathrm{R}), \mathrm{R} \text { in kilo- } \\
\text { meters }\end{array}$ \\
\hline Penetration Loss & $10 \mathrm{~dB}$ \\
\hline \multicolumn{2}{|l|}{ Log Normal Fading } \\
\hline Standard Deviation & $8 \mathrm{~dB}$ \\
\hline Inter-NodeB Correlation & 0.5 \\
\hline Intra-NodeB Correlation & 1.0 \\
\hline Correlation Distance & $50 \mathrm{~m}$ \\
\hline Max BS Antenna Gain & $14 \mathrm{dBi}$ \\
\hline \multirow{2}{*}{$\begin{array}{l}\text { Antenna pattern } \\
\text { 2D Pattern }\end{array}$} & \\
\hline & $\begin{array}{l}A(\theta) \\
-\min \left(12\left(\theta / \theta_{3 d B}\right)^{2}, A m\right), \text { where } \\
\theta_{3 d B}=70 \text { degrees }, A m=20 d B\end{array}$ \\
\hline Channel Model & PedA $3 \mathrm{~km} / \mathrm{h}$ \\
\hline CPICH Ec/Io & $-10 \mathrm{~dB}$ \\
\hline Total Overhead power & $30 \%$ \\
\hline UE Antenna Gain & $0 \mathrm{dBi}$ \\
\hline UE noise figure & $9 \mathrm{~dB}$ \\
\hline UE Receiver Type & Type3i with $2 \mathrm{Rx}$ antennas \\
\hline Spreading factor & 16 \\
\hline $\begin{array}{l}\text { Maximum Sector } \\
\text { Power }\end{array}$ & $43 \mathrm{dBm}$ \\
\hline Handover reporting threshold & $6 \mathrm{~dB}$ \\
\hline Multiflow operation & Inter- and intra-site \\
\hline Number of HARQ processes & 6 \\
\hline Number of UEs/cell & $\begin{array}{l}1,2,4,8,16 \text { and } 32 \text {. UEs dropped } \\
\text { randomly across the system }\end{array}$ \\
\hline Traffic & \\
\hline Traffic model & Bursty Traffic Source Model \\
\hline File size & Fixed at $1 \mathrm{Mbit}$ \\
\hline File inter-arrival time & Exponential, mean 5 seconds \\
\hline Flow control on Iub & Ideal and instantaneous \\
\hline HS-DPCCH Decoding & $\begin{array}{l}\text { ACK decoded on UL; C2P used: } 2 \\
\text { dB for Intra-NB, } 4 \mathrm{~dB} \text { for Inter-NB }\end{array}$ \\
\hline CQI & Ideal with 3 TTI delay \\
\hline
\end{tabular}

area. The used bursty FTP traffic model has a constant file size but the file inter-arrival time for a UE follows exponential distribution with a mean value of 5 seconds. Each UE has an own traffic generator with an independent arrival probability.

A Multiflow capable UE that is located at the cell border may receive transport blocks from two cells. For modeling purposes this type of UE may be divided into two conceptual entities which are independent from each other: class A UE entity that is served by the strongest (primary) cell and class B UE entity that is served by the second strongest (secondary) cell. The received amount of data is aggregated from both of these entities in the end of the simulation so as to attain the total amount of data received by a single Multiflow user. Although each UE has an independent data buffer, class A and B Multiflow entities share a common traffic generator so as not to have doubled amount of files for one Multiflow UE.

Following the user entity division, the basic proportional fair scheduling (see e.g. [10]) is extended for Multiflow regarding the transmit opportunities from the secondary cell to class B UE entity. In case the Multiflow UE is in soft or softer handover area, the class B UE is scheduled only if there are no active class A UEs in that cell. This results in reduced usage of Multiflow when the load level of the network increases. It is observed that Multiflow can provide gains until the network load corresponds to virtually user full-buffer traffic, a rare situation in real HSDPA networks. Class A UEs are treated normally as other non-Multiflow UEs in each cell.

A $6 \mathrm{~dB}$ handover margin is used which results approximately in $9 \%$ and $36 \%$ UE percentages in softer handover (softer $\mathrm{HO}$ ) and soft handover (SHO) area, respectively. In our simulations, all of these UEs are Multiflow capable. It has been noted by different studies that legacy UEs who are not capable of receiving two data flows concurrently and who may not use interference aware receivers do not suffer from Multiflow, thus for simplification they are left out from our tests (see e.g. [11][12])

We assume ideal time and frequency synchronization of Multiflow transmissions regardless whether the cells are controlled by the same or different NodeBs. Also the Doppler shift is assumed similar. The $I_{u b}$ interface flow control is ideal as both cells participating Multiflow acquire bits from a common user-specific buffer of data that is comprised of queued files.

\section{A. Simulation Results}

Fig. 2 presents cumulative distribution function (CDF) of UE burst rates (throughputs) for the case where on average eight UEs are present in each cell in the network. Total amount of UEs in the network is thus $57 \times 8=456$. These curves include results from all UEs in the network. In the figure, the "Reference" curve displays the results where Multiflow was switched off and UEs can receive data only from their primary cell. The curve labeled "Multiflow" show the case where Multiflow was enabled and the UEs within the HO margin may receive transmissions over both primary and secondary link. Fig. 3 provides separated results for three user terminal groups: users in the soft handover area (i.e. users who reside at the border of two cells served by different NodeBs), users in the softer handover area (i.e. users who reside at the border of two cells served by the same NodeB) and non-HO users who do not reside at cell borders.

The first observation from Fig. 2 is that low and medium burst rate UEs achieve biggest gain when using Multiflow, which is due to the fact that many of these UEs reside in the handover region and therefore may utilize two data flows from two cells. The burst rate distribution can be easily seen from Fig. 3 by looking at the burst rates of softer $\mathrm{HO}$ and $\mathrm{SHO}$ area UEs. On average, UEs in SHO region have the lowest burst rate among the groups due to high inter-site interference and long distance to their serving cell site, and softer $\mathrm{HO}$ area UEs achieve mostly low or medium burst rates. Therefore, gains for high burst rate UEs stay at a very low level. Thus the throughput fairness across UEs is enhanced.

By evaluating the Multiflow gains from Fig. 3, it is evident that handover area UEs in all burst rate levels levels have equally gained from receiving assistance on the secondary link. An important characteristic of Multiflow is that a large pool of UEs is benefiting in equal manner. By increasing 


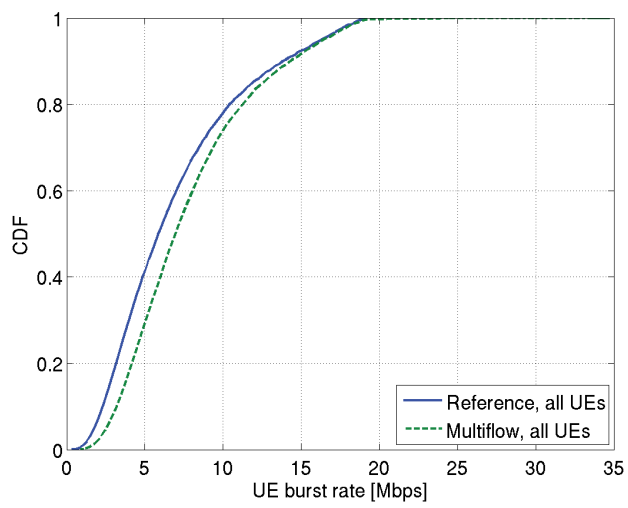

Fig. 2. Burst rate CDF of all UEs, 8 UEs/cell

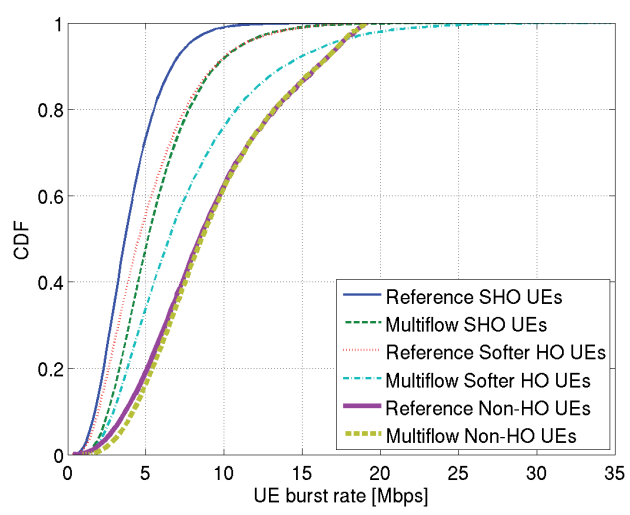

Fig. 3. Burst rate CDF of $\mathrm{HO}$ and non-HO area UEs, 8 UEs/cell

the handover threshold margin the pool could be even larger, however defining too large a margin would result in reduction of Multiflow performance due to increased imbalance between the primary and secondary link. Also, the UL control signaling would need to be dimensioned to be reliably received by both cells. Equivalently, having smaller margin allows the link balance to approach 1:1 ratio for all handover area users, but with a cost of a smaller user pool size.

Fig. 3 also presents burst rates for non-handover UEs who usually reside closer to cell center or cell site and will not be able to receive Multiflow transmissions from two cells, but who are served normally by a single cell. As seen from the figure, there is no negative impact from Multiflow to these UEs, and even a slight improvement is visible. Although Multiflow does not affect the non-HO area UEs directly, the implicit impact can be both negative and positive. Total interference in the network might be increased as Multiflow UEs may activate otherwise empty cells (negative impact).

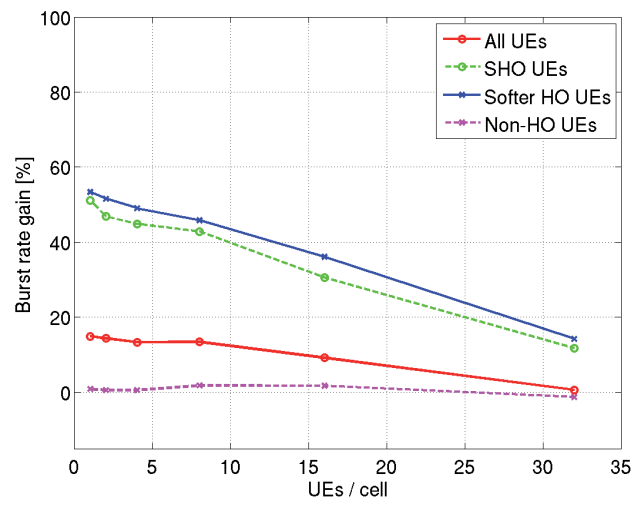

Fig. 4. Burst rate gains for various load levels

However, improved burst rates and by that reduced activity times of Multiflow UEs allow more scheduling opportunities for all other UEs, which affects positively to burst rates of non-HO area terminals.

Percentual burst rate gains for all simulated load levels are shown in Fig. 4. UEs in handover region, either in softer $\mathrm{HO}$ or SHO, have high gains starting from over $50 \%$, then decreasing towards high loads. The degradation is caused by fewer opportunities to schedule a transmission on the secondary link since there is a higher probability for a cell to have active class A UEs of its own as the number of UEs per cell increases.

One should notice that the burst rate gain for non- $\mathrm{HO}$ area UEs stays close to $0 \%$, demonstrating that there is no overall negative impact from Multiflow. This is ensured by the nonblocking scheduling operation where class A UEs are favored over class B UEs.

Absolute mean burst rates for all UEs in the simulations are presented in Fig. 5. This is an alternative representation of the burst rate gains for all UEs in the network displayed in Fig. 4, but in addition showing that a larger number of UEs results in extended UE activity times and thus decreased absolute data rates.

To support and explain the burst rate gains presented in Fig. 4, cell and user activity ratios are gathered in Table II. The cell TTI usage numbers provide information on how many TTIs cells transmit data during the simulations, i.e.

$$
\text { cellTTIusage }[\%]=\frac{\text { Num }}{\text { activeTTIs }} \times 100 .
$$

The numbers are gathered from both reference and Multiflow simulations. The table contains also activity statistics for Multiflow users, separated into activity levels of primary and secondary link. These are the ratios between the number of TTIs when users are scheduled over the number of TTIs when users could be scheduled, i.e. when there is data for those users in the transmission buffers. It can be observed that for low 


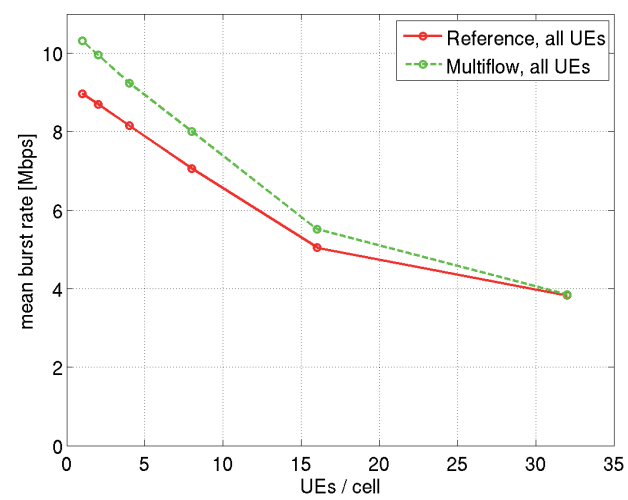

Fig. 5. Mean burst rates for various load levels TABLE II

TTI ACTIVITY

\begin{tabular}{|c|c|c|c|c|}
\hline & \multicolumn{2}{|c|}{ Cell TTI usage [\%] } & \multicolumn{2}{c|}{ Multiflow user TTI activity [\%] } \\
\hline UEs/cell & Reference & Multiflow & Primary link & Secondary link \\
\hline 1 & 4.4 & 4.3 & 97.9 & 96.5 \\
\hline 2 & 6.6 & 7.2 & 95.9 & 92.8 \\
\hline 4 & 12.0 & 14.4 & 91.7 & 84.8 \\
\hline 8 & 25.6 & 31.2 & 81.5 & 68.0 \\
\hline 16 & 59.6 & 68.3 & 54.9 & 30.7 \\
\hline 32 & 97.6 & 99.6 & 9.7 & 0.2 \\
\hline
\end{tabular}

loads cells remain silent over $90 \%$ of the time, and Multiflow users can be scheduled almost always over both primary and secondary link. The average cell activity level in the Multiflow simulations is higher than in the reference simulations, which is expected as two flows can be utilized instead of one. Also in the user activity statistics we see that the utilization rate of the secondary link declines towards higher loads, as expected. However, even for moderately high load, such as 8 or 16 UEs per cell, there is still a high probability that the secondary cell will not serve its class A UEs, and therefore could schedule a class B UE. Indeed, by looking at Fig. 4, the gains (for all UEs) approach zero only for 32 UEs per cell when the network offered load leads to UEs having very few transmission opportunities and effectively having full-buffer traffic. However, some gains are still visible for handover area terminals.

\section{CONCLUSION}

In this paper an HSDPA multi-cell transmission scheme called Multiflow is presented. A discussion on the requirements and solutions for the concept is provided together with simulation results gathered with semi-static network simulations.

In Multiflow, two sectors under the control of one or two NodeBs transmit independent data blocks to a user. To support Multiflow, user terminal equipment requires an interference aware type $3 i$ receiver with spatial diversity in order to reduce the interference between the two received flows. Enhanced flow control is needed in the network as well as data splitting to each participating cell before the transmission in either the Radio Network Controller or the NodeB. Data combining is alike necessary at the user terminal when merging the flows back together.

The simulation results show that Multiflow provides substantial gains of around for $40 \%$ for cell edge users who otherwise may reach only low or mediocre data rates. The gains are realized primarily by utilizing free transmission resources in neighboring cells, amounting to short-term load balancing. This directly results in improved mean user throughput as the users may finish their data transmissions and leave the network earlier, which in part helps other existing users by releasing more resources for them. Although Multiflow may create additional interference to the network, the advantages from the concept are predominant.

\section{ACKNOWLEDGMENT}

The authors would like to thank Petri Jolma and Jani Moilanen from Nokia Siemens Networks for their valuable contributions to the Multiflow work. Also special thanks to Michal Maternia, Krystian Pawlak and Patrick Marsch from Nokia Siemens Networks for their assistance with the development of the network simulator.

\section{REFERENCES}

[1] 3GPP TSG-RAN, RP-111375, "HSDPA multiflow data transmission," Sep 2011.

[2] 3GPP TR 36.814 v9.0.0, "Further advancements for E-UTRA physical aspects," Mar 2010

[3] Minghai Feng, Xiaoming She, Lan Chen and Yoshihisa Kishiyama, "Enhanced Dynamic Cell Selection with Muting Scheme for DL CoMP in LTE-A," in Vehicular Technology Conference Spring, 2010, Taipei, Taiwan, May 2010

[4] Binbin Wang, Bingbing Li and Mingqian Liu, "A Novel Precoding Method for Joint Processing in CoMP," in Network Computing and Information Security, 2011, Guilin, China, May 2011.

[5] T. Nihtilä, J. Kurjenniemi and E. Virtej, "System Level Analysis of Interference Aware LMMSE Chip Equalization in HSDPA Network," in IEEE Symposium on Computers and Communications, 2007. ISCC 2007, Aveiro, Portugal, Jul 2007, pp. 133-138.

[6] 3GPP TSG-RAN WG1, R1-111141, Nokia Siemens Networks, Nokia, "Multiflow performance evaluation," Feb 2011

[7] D.M. de Andrade, A. Klein, H. Holma, I. Viering and G.Liebl, "Performance Evaluation on Dual-Cell HSDPA Operation," in Vehicular Technology Conference Fall, 2009, Anchorage, USA, Sep 2009.

[8] 3GPP TSG-RAN WG1, R1-111775, Nokia Siemens Networks, Nokia, 3GPP TSG-RAN WG1, R1-111775, Nokia Siemens Networks, Nokia,
"Signaling and configuration for the multi-point transmission schemes," "Signaling
May 2011.

[9] 3GPP TSG-RAN, R1-111116, TR 25.872, V0.1.3, "HSDPA multipoint transmission," Mar 2011

[10] A. Jalali, R. Padovani and R. Pankaj, "Data throughput of CDMA-HDR a high efficiency-high data rate personal communication wireless system," in Vehicular Technology Conference Proceedings, 2000, Tokyo, Japan, May 1997, pp. 1854-1858.

[11] 3GPP TSG-RAN WG1, R1-111539, OUALCOMM Incorporated, "System Performance Evaluation of SF-DC Inter NodeB Aggregation in tem Performance Evaluation of SF-DC Inter NodeB Aggregation in
Uniform Loading Scenario Assuming Realistic RLC and Flow Control (30\% Penetration)," May 2011

[12] 3GPP TSG-RAN WG1, R1-112627, Nokia Siemens Networks, Nokia, "Text proposal on the performance of multiflow (SF-DC aggregation) in legacy deployments (30\% multiflow penetration)," Aug 2011. 